\title{
Percutaneous Endoscopic Interlaminar Discectomy for L5-S1 Adolescent Lumbar Disc Herniation
}

\author{
Zheng XU, Yi LIU, Jinchuan CHEN \\ Xuzhou Medical University, Affiliated Hospital of Lianyungang, Department of Orthopedics, Lianyungang, China
}

\section{ABSTRACT}

AIM: L5/S1 Adolescent lumbar disc herniation (ALDH) is usually ignored for its low incidence. Recently, percutaneous endoscopic procedures have been widely used. To investigate the surgical techniques and clinical outcomes of percutaneous endoscopic interlaminar discectomy (PEID) in the treatment of adolescent L5-S1 disc herniation (ALDH).

MATERIAL and METHODS: A retrospective study was performed between May 2012 and September 2014, which included 23 adolescent patients who underwent PEID for L5/S1 lumbar disc herniation. Ten females and 13 males were included, with a mean age of 15.4 years and a mean BMI of $25.1 \mathrm{~kg} / \mathrm{m}^{2}$. The pre- and postoperative visual analogue scale (VAS), Japanese Orthopaedic Association (JOA), and modified MacNab scores, as well as perioperative data, were collected.

RESULTS: The mean follow-up period was 19.7 months. The average operation time and hospital stay were $80.9 \pm 7.3$ minutes and $5.8 \pm 1.2$ days, respectively. During the follow-up period, the VAS for back and leg pain decreased significantly, and JOA scores greatly increased $(p<0.05)$. In terms of modified MacNab criteria, $86.9 \%$ of patients showed excellent or good results. After surgery, two patients complained of leg numbness, and another patient underwent a subsequent open discectomy due to recurrent disc herniation.

CONCLUSION: Considering specific anatomical peculiarities and expectation for rapid recovery, PEID is a safe and effective minimally invasive technique for ALDH at the L5-S1 level.

KEYWORDS: Adolescent, L5-S1 level, Lumbar disc herniation, Percutaneous endoscopic interlaminar discectomy

\section{INTRODUCTION}

$\mathrm{L}$ umbar disc herniation (LDH) is a common spinal disorder in adults. However, the prevalence of patients aged less Ithan 20 years, who were defined as adolescent lumbar disc herniation (ALDH), was very low, particularly at the L5S1 level $(12,13)$. Surgical treatment should be considered if a favorable outcome cannot be achieved after conservative therapy. Open discectomy was used to cure most adult lumbar disc herniation cases, but several complaints, such as residual low back pain, temporary leg anaesthesia, or long hospital stay have been increasing, indicating that open discectomy is not the preferred surgical treatment $(11,21)$. In addition, adolescents showed developing skeletons and specific spino-pelvic sagittal alignment during rapid growth (5). These characteristics may result in further consideration on surgical techniques for ALDH.

With the popularity of minimally invasive surgical technique, percutaneous endoscopic procedures for ALDH yielded good results, similar to those with open discectomy. Compared with open discectomy, percutaneous endoscopic procedures can preserve normal spinal structures, such as ligaments, muscle, lamina and facet joints, minimizing the effects on growth of adolescence $(3,21)$. There are two major procedures, percutaneous endoscopic transforaminal discectomy (PETD) and percutaneous endoscopic interlaminar discectomy (PEID), according to different approaches. Previous studies 
have proven PETD to be an effective option for ALDH $(19,20)$. However, the most commonly involved level in ALDH is L45, and L5-S1 ALDH has been rarely reported. Moreover, the transforaminal approach to L5-S1 disc space is narrowed due to a high iliac crest and a large $L 5$ transverse process, and obviously PETD is not the first selection for L5-S1 ALDH. Under such conditions, the emphasis of the present study was to evaluate the surgical outcome of PEID in 23 consecutive ALDH patients.

\section{MATERIAL and METHODS}

This retrospective study consisted of 23 consecutive adolescent patients who underwent PETD for L5-S1 ALDH from May 2012 to September 2014. The inclusion criteria were as follows:

(1) Patients $\leq 21$ years old

(2) The affected level was limited in the L5/S1 level on the computed tomography (CT) scan or magnetic resonance imaging (MRI)

(3) Patients showed symptoms that were consistent with radiological findings

(4) No obvious improvement was achieved after conservative therapy at least 2 months. Patients with intraspinal neoplasm, lumbar tuberculosis, congenital deformity or segmental instability were excluded. Patients with organic diseases were also excluded.

There were 10 females and 13 males, with a mean age of 15.4 years (range: $10-21$ years) and a mean BMl of $25.1 \mathrm{~kg} /$ $\mathrm{m}^{2}$ (range: $18.4-32.6 \mathrm{~kg} / \mathrm{m}^{2}$ ). Twenty patients (86.9\%) suffered from low back pain and leg pain. Three patients $(13.0 \%)$ complained of leg pain only. Eighteen patients $(78.2 \%)$ presented with positive straight leg-raising test. Fourteen patients $(60.9 \%)$ showed leg motor weakness (power grade III to IV). Nine patients (39.1\%) showed a forward-bending posture by lower limb inflexion while standing.

Clinical manifestations and radiographical outcomes were analysed. Perioperative events were recorded. We compared the preoperative visual analogue scale (VAS) (26), and Japanese Orthopaedic Association (JOA) scale (6), with those at 1-, 6-, 12- and 18-month follow-ups. The modified MacNab criteria at the last follow-up were confirmed via questionnaires (18).

Continuous variables were presented as the mean $\pm S D$. Statistical analysis was performed with a paired Student's t-test. SPSS Ver. 22 (SPSS Inc. Chicago, IL, USA) software was used. Two-tailed $p<0.05$ was considered statistically significant.

\section{Surgical Technique}

All patients were operated under general anaesthesia. After preoperative intravenous antibiotic, patients were placed on a radiolucent table in the prone position, and the spine was flexed to increase the interlaminar window. Electromyogram was connected to patients, monitoring any nerve stimulation, and determined if the compression should be removed. Under anteroposterior film, the midline was marked along the tips of the spinous processes at L5-S1. The horizontal line was also marked between the superior endplate of S1 lamina and the inferior endplate of L5 lamina. The skin entry point was approximately $1.0 \mathrm{~cm}$ lateral to the intersection of the above two lines. The axillary type of L5-S1 adolescent disc herniation was obtained. Next, the spine needle was inserted vertically and slightly below the interlaminar centre, along the lateral edge of the interlaminar window (Figure 1A). Once the facet joint of S1 was touched, we inserted the guide wire along the spine needle, and the needle was changed to dilators from 1 to $7.5 \mathrm{~mm}$ in diameter. Indigo carmine was used to dye the protruded nuclear tissue blue. These procedures were monitored by anteroposterior and lateral radiographs to ensure that the dilators and indigo carmine were placed correctly and at the proper depth (Figure 1B).

After the working cannula was inserted over the dilators (Figure 1C), the endoscope (OLYMPUS, Japan) with a continuous inflow saline was introduced. Through endoscopic vision, the ligamentum flavum and epidural fat were the first structure to encounter. Due to their yellow color, the ligamentum flavum and epidural fat were easily identified and then carefully removed by different graspers. The herniated disc fragments were exposed with blue stain. In addition, the bipolar radiofrequency electrode could also help to regulate haemostasis and dissect disc fragments. We rotated the working cannula to further clean residual disc tissues at the shoulder region. Under direct visual control, the scope with the cannula was gradually removed until the pulsing S1 root was observed. For ALDH, we felt that it was not necessary to anchor the cannula into the disc space to induce further depression because the disc tissues were still in growth, and too early destruction might lead to spinal instability and developmental disorder. Finally, a single stitch was given to close the incision.

\section{RESULTS}

All 23 L5-S1 ALDH patients underwent PETD smoothly and followed-up with an average period of 19.7 months. According to preoperative radiology, all 23 ALDH showed L5-S1 herniation, and 15 (65.2\%) patients exhibited lower T2 signal intensity L5-S1 disc.

The mean operation time was $80.9 \pm 7.3$ minutes. All patients except three patients showed relief of their symptoms after the operation. Two patients complained of unilateral leg numbness, which disappeared gradually after 1 month without any intervention. Stretch injury of the nerve root during operation may be a main factor. Another patient diagnosed with endplate fracture underwent a subsequent open discectomy due to recurrent disc herniation that had occurred after 6 months. The VAS scores for back and leg pain decreased greatly at the 1 -month follow-up $(p<0.05)$ (before operation: $6.2 \pm 1.3$; at 1-month: $2.3 \pm 1.2$ ) and remained stable at 6-,12- and 18-month follow-ups (p>0.05) (at 6-month: $1.8 \pm$ 0.9 ; at 12-month: $1.4 \pm 0.7$; at 18 -month: $1.2 \pm 0.6$ ). The JOA scores kept increasing until the last follow-up $(p<0.05)$ (before 


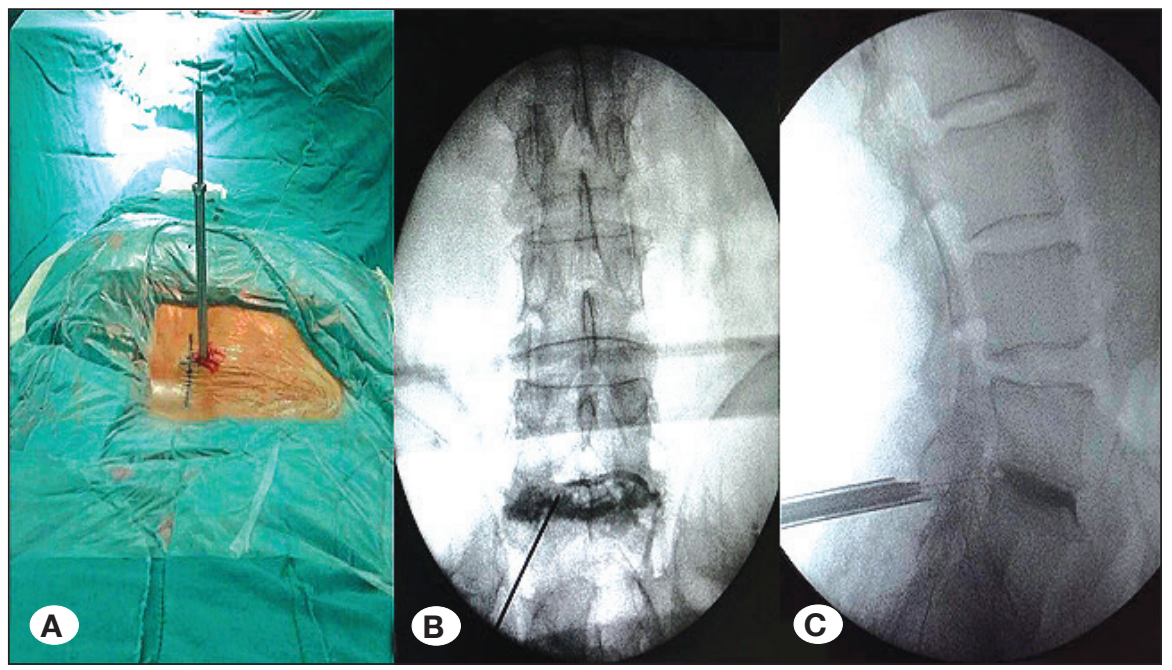

Figure 1: Procedures of PEID. A) The guide wire and working cannula was vertically inserted into L5/S1. B) Anteroposterior view of the guide wire inserted into the L5/S1 interlaminar space. C) Lateral view of the working cannula inserted over the guide wire into the L5/S1 interlaminar space.

operation: $12.5 \pm 3.8$; at 1 -month: $23.7 \pm 2.8$; at 6 -month: $25.3 \pm 2.4$; at 12 -month: $26.6 \pm 1.9$; at 18-month: $27.6 \pm 1.3$ ) (Figures 2, 3). The modified MacNab criteria at the last followup were excellent for 18 patients $(78.2 \%)$, good for 2 patients $(8.7 \%)$, and fair for 3 patients (13.0\%), and no poor results were observed. There were no cases of infection or dural tear in the present study. A typical case is shown in Figure 4A-D.

\section{DISCUSSION}

ALDH is a rare disease with an incidence of only $1 \%-5 \%$ $(7,12,13)$. Since Wahren reported the first case of ALDH in a 12-year-old child (25), concerns on the specific subgroup of LDH have increased. However, the mechanism of ALDH remains controversial. According to previous studies $(14,21)$, age-driven proteoglycan loss with subsequent increasing collagen leads to a protruded disc, which may be attributed to an uneven distribution of the axial load and accelerated disc degeneration. Thus, it was suggested that lumbar degeneration is the main risk factor of LDH in elderly people. Boos et al. noted that disc degeneration most likely begins in childhood (1), although no explanation yet exists. In our study, according to the MRI of all patients, 15 cases $(65.2 \%)$ showed lower T2 signal intensity of the L5/S1 intervertebral disc, which confirmed the above viewpoints that disc degeneration could be observed at an early age. In recent years, with heavier homework and a lack of exercise, unhealthy life styles have spread among young people, leading to an increasing rate of the obesity in youth. A strong association between body mass index (BMI) and obesity has been documented (23). The WHO defined overweight as a BMI of $25-29.9 \mathrm{~kg} / \mathrm{m}^{2}$ and obesity as a BMl of $30 \mathrm{~kg} / \mathrm{m}^{2}$ or greater (24). In 15 patients with lower T2 signal intensity, we found that 10 patients $(66.7 \%)$ showed a BMI of $>25 \mathrm{~kg} / \mathrm{m}^{2}$, and 3 patients (20\%) showed a BMI of $>30 \mathrm{~kg} / \mathrm{m}^{2}$. To the best of our knowledge, hours spent sitting and obesity can result in muscle fatigue of facet joints, which in a vicious circle may potentially cause further disc degeneration. Thus, early disc degeneration may be a nonnegligible factor of ALDH. Endplate fracture was considered to be another important factor of ALDH. Adolescents have

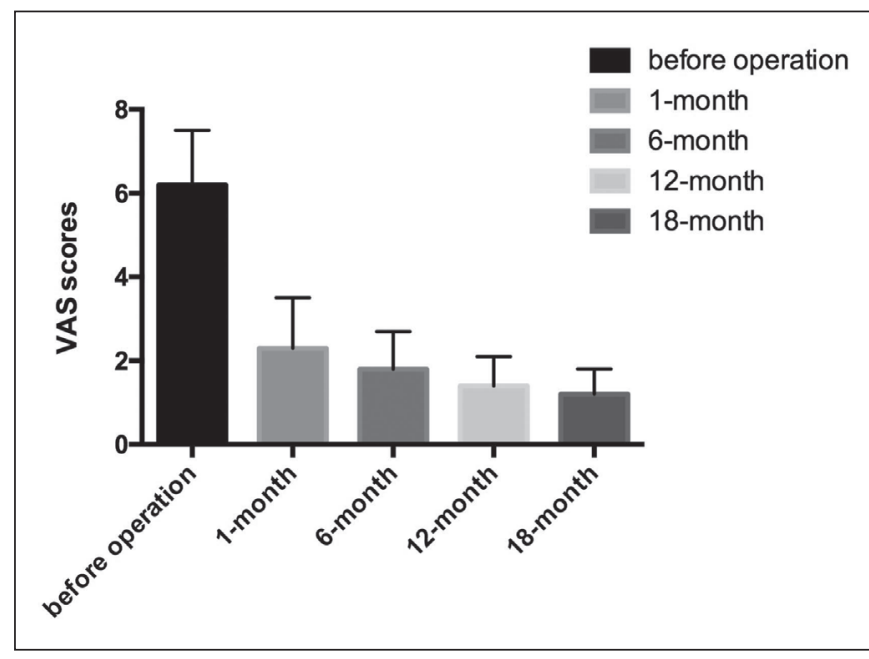

Figure 2: VAS scores before and after the operation.

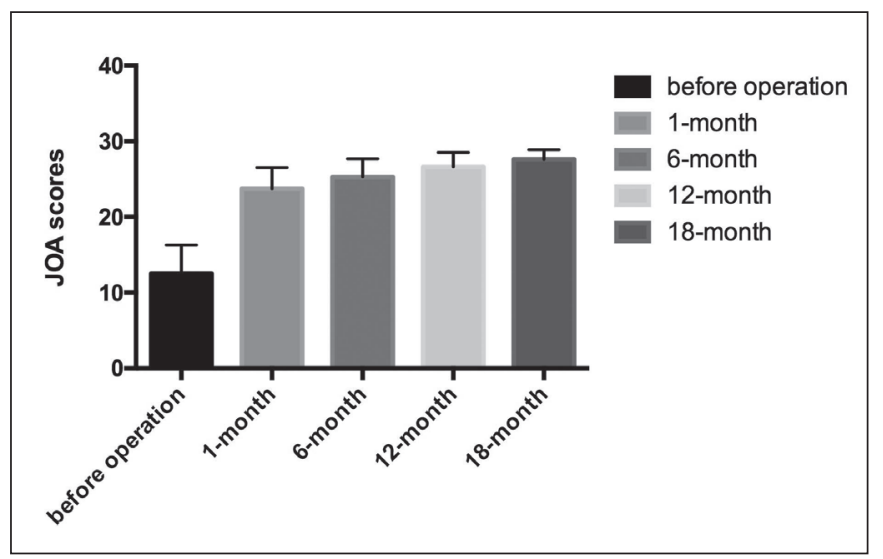

Figure 3: JOA scores before and after the operation. 

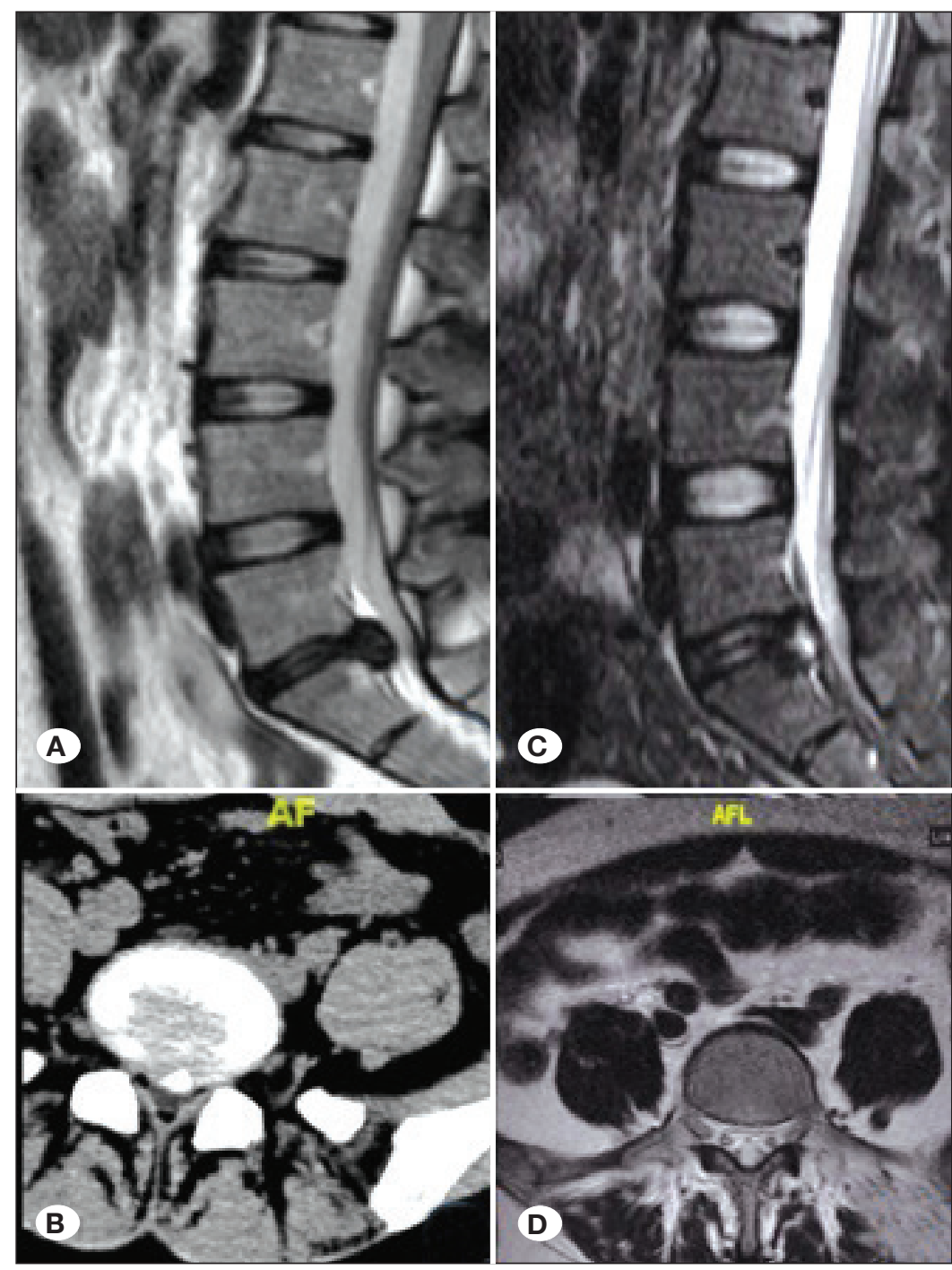

Figure 4: Imaging examinations of a 14-year-old female L5-S1 ALDH before and after PEID.

A) Sagittal MRI of the patient before PEID.

B) Transaxial CT scan of the patient with disc herniation to the left side before PEID.

C) Sagittal MRI of L5-S1 herniated nucleus pulposus removed after PEID at 12 months. D) Transaxial MRI of L5-S1 herniated nucleus pulposus removed after PEID at 12 months.

a strong predisposition for lumbar disc herniation by trauma due to frequent outdoor activities, and the trauma is a causal factor for endplate fracture $(9,13)$. There are two mechanisms of trauma proposed for these lesions: hyperextension of the lumbar spine and rapid flexion together with axial compression to the vertebral column (9). In our research, only $2(8.7 \%)$ patients had a history of significant trauma and were diagnosed with endplate fracture radiologically. Compared with 15 patients $(65.2 \%)$ showing degenerative changes, the lower incidence of trauma led to the assumption that gross trauma may not be a primary factor, which is consistent with the results obtained by Kumar et al. (10).

Endo et al. compared the spinal parameters of LDH in adults and healthy people and concluded that the loss of lumbar lordosis resulted in an anterior displacement of C7 plumb line (5). In our study, we found that 9 ALDH patients showed a forward-bending posture by lower limb inflexion while standing and complained of lower back pain. We assumed that these abnormal postures might be secondary to an analgesic response to avoid sciatic pain. Due to a lack of lateral wholespine standing radiographs, ALDH spinal parameters cannot be measured. Further study about the relationship between ALDH and sagittal spinal alignment should be identified.

PETD used in L4-5 ALDH has matured over the past several years (15), but research on microdiscectomy used for L5-S1 ALDH has been rarely reported. The present study is focus on minimally invasive surgery for L5-S1 ALDH. Adolescents are in the golden period in terms of growth and the accumulation of knowledge. Parents and children both fully expect higher postoperative life quality. The outcome of lumbar discectomy is dependent on the surgical technique (17). Thus, an effective surgical technique is necessary for young people. It is almost inevitable for open surgery to damage paraspinal muscles and other normal tissues, which start to develop when 10 years old until maturation at approximately 25 years (22). These injuries affect adolescent growth as well as increase the risk of lumbar instability. Furthermore, slower recovery and longterm brace treatment may delay the progression of the study. With the improvement in optics and the rod lens system, the percutaneous endoscopic technique is a good option for ALDH patients with preserved muscles and an early return to school $(8,16)$. 
Unlike L4-5, L5-S1 has its own anatomical peculiarities. First, the existence of a high iliac crest and a large L5 transverse process has limited the transforaminal approach to the L5-S1 disc space. Second, through cadaveric dissection, Ebraheim et al. found that the foraminal diameter was the narrowest at the L5-S1 level (average, $13.4 \mathrm{~mm} \pm 4.1 \mathrm{~mm}$ ) (4), which was notably below the average of whole levels (average, $24 \mathrm{~mm}$ $\pm 3 \mathrm{~mm}$ ). Third, the joint facets at the L5-S1 level overlaps with the disc space and isthmus of L5, which extends more laterally to hide the waist of the vertebral body. These anatomical characteristics all limit the transforaminal access to the L5-S1 disc space, which indicates that PETD is not applicable for L5-S1 ALDH. In the present study, we adopted another technique-PEID and recorded VAS and JOA scores at every follow-up point. The modified MacNab criteria were calculated at the last follow-up. We found an improvement of VAS and JOA, which were significantly different from those in preoperation, suggesting that PEID would be beneficial for L5-S1 ALDH to relieve pain. The excellent and good portion in MacNab accounted for $86.9 \%$, which could also explain the good results.

In contrast, from PETD, PEID is a minimally invasive technique that can directly reach the spinal canal via the interlaminar space (20). Ebraheim et al. confirmed that the interlaminar distance was largest at L5-S1 (4). Specifically, in ALDH with forward-bending posture, the interlaminar distance was relatively greater. Moreover, the spinal canal at L5-S1 demonstrates the largest volume such that more free space is given (2). In addition, the ligamentum flavum of the L5-S1 interlaminar space is the thinnest compared to any other level (2), and its thickness is associated with age-related changes; the younger, the thinner. These anatomical features make it easy to access the plane of the disc using instruments without injury to the thecal sac and S1 nerve root.

During PEID, attention should be paid to several aspects.

1. The S1 root is usually compressed in L5-S1 LDH, while the S1 root extends from the thecal sac in parallel, overlapping the disc of L5-S1, such that it is possible to injure the S1 root when accessing the herniations through a cannula. Before entering the intraspinal space, we performed serial dilatation over the guide wire with dilators starting from 1 to $7.5 \mathrm{~mm}$ in diameter, which can expend our vision and prevent nerve injury. Before surgery, we must identify whether the herniation is at the axillary or shoulder region using CT or MRI. In our study, there were two patients with S1 root contusion, who complained of transient dysesthesia after surgery. One case occurred because the preoperative CT cross-sectional images were ignored. Another case occurred because of strenuous stretching. Most cases of ALDH are contained lumbar disc herniation that is featured as largeness, tenacious and even dislocated. Thus, it is not easy for an inexperienced surgeon to remove the targets. Rough handling is inevitable. Thus, careful and slow operations are key to obtaining successful results.

2. Currently, local anaesthesia has been recommended in PIED, which can help surgeons to obtain an immediate reaction if the nerve root is mistakenly stimulated. However, in our study, PEID was performed under general anaesthesia to avoid pain from repeated punctures. An electromyogram must be used to monitor whether the nerve root is damaged.

3. For adolescents diagnosed with endplate fracture, PEID appeared less effective. In our study, 2 patients with endplate fractures were operated by PEID; however, the cannula cannot easily detour around the spinal cord, and herniation that has settled ventrally from the spinal canal is very difficult to completely remove. One of the patients underwent a subsequent open discectomy because recurrent disc herniation occurred after 6 months.

Thus, we suggested that PEID should be further considered for adolescents with a specific diagnosis of endplate fracture.

\section{- CONCLUSION}

ALDH is a rare disorder with an inconclusive mechanism. To prevent $\mathrm{LDH}$ at an early age, healthy diets and outdoor exercises have been proposed to be beneficial. In consideration of specific anatomic peculiarities and expectation for rapid recovery, PEID is a safe and effective minimally invasive technique for ALDH at the L5-S1 level.

\section{ACKNOWLEDGEMENT}

We thank all those who participated in the study. The work has been supported by Department of Orthopedics, Xuzhou Medical University Affiliated Hospital of Lianyungang in China.

\section{REFERENCES}

1. Boos N, Weissbach S, Rohrbach H, Weiler C, Spratt KF, Nerlich AG: Classification of age-related changes in lumbar intervertebral discs: 2002 Volvo Award in basic science. Spine (Phila Pa 1976) 27: 2631-2644, 2002

2. Choi G, Lee SH, Raiturker PP, Lee S, Chae YS: Percutaneous endoscopic interlaminar discectomy for intracanalicular disc herniations at L5-S1 using a rigid working channel endoscope. Neurosurgery 58: ONS59-68; discussion ONS59-68, 2006

3. Dabo X, Ziqiang C, Yinchuan Z, Haijian N , Kai C, Yanbin L, Qiang F, Chuanfeng W: The clinical results of percutaneous endoscopic interlaminar discectomy (PEID) in the treatment of calcified lumbar disc herniation: A case-control study. Pain Physician 19: 69-76, 2016

4. Ebraheim NA, Xu R, Huntoon M: Location of the extraforaminal lumbar nerve roots. An anatomic study. Clin Orthop Relat Res 340: 230-235, 1997

5. Endo K, Suzuki H, Tanaka H, Kang Y, Yamamoto K: Sagittal spinal alignment in patients with lumbar disc herniation. Eur Spine J 19: 435-438, 2011

6. Fukui M, Chiba K, Kawakami M, Kikuchi S, Konno S, Miyamoto M, Seichi A, Shimamura T, Shirado O, Taguchi $\mathrm{T}$ : Japanese Orthopaedic Association cervical myelopathy evaluation questionnaire (JOACMEQ): Part 2. Endorsement of the alternative item. J Orthop Sci 12: 241-248, 2007 
7. Haeusler M, Schiess R: Evidence for juvenile disc herniation in a homo erectus boy skeleton. Spine (Phila Pa 1976) 38: E123128,2013

8. Kambin P: Arthroscopic microdiscectomy: An alternative to open disc surgery. Mt Sinai J Med 67: 283-287, 2000

9. Keller RH: Traumatic displacement of the cartilagenous vertebral rim: A sign of intervertebral disc prolapse. Radiology 10: 21-24, 1974

10. Kumar R, Kumar V, Das NK, Behari S, Mahapatra AK: Adolescent lumbar disc disease: Findings and outcome. Childs Nerv Syst 23: 1295-1299, 2007

11. Kwon DW, Kim KH, Park JY, Chin DK, Kim KS, Cho YE, Kuh SU: Clinical outcomes and considerations of the lumbar interbody fusion technique for lumbar disk disease in adolescents. Childs Nerv Syst 29: 1339-1344, 2013

12. Lavelle WF, Bianco A, Mason R, Betz RR, Albanese SA: Pediatric disk herniation. J Am Acad Orthop Surg 19: 649656, 2011

13. Lee JY, Ernestus RI, Schröder R: Histological study of lumbar intervertebral disc herniation in adolescents. Acta Neurochir (Wien) 142:1107-1110, 2000

14. Lian XF, Hou TS, Xu JG, Zeng BF, Zhao J, Liu XK, Yang EZ, Zhao C: Single segment of posterior lumbar interbody fusion for adult isthmic spondylolisthesis: Reduction or fusion in situ. Eur Spine J 23: 172-179, 2014

15. Maroon JC: Current concepts in minimally invasive discectomy. Neurosurgery 51: S137-145, 2002

16. Mayer HM, Mellerowicz H: Endoscopic discectomy in pediatric and juvenile lumbar disc herniations. J Pediatr Orthop B 5: 3943, 1996

17. McCulloch JA: Focus issue on lumbar disc herniation: Macroand microdiscectomy. Spine (Phila Pa 1976) 21: 45S-56S, 1996
18. McCulloh JA: The lumbar spine-small is beautiful: The Third Annual Ian Macnab Memorial Lecture. J Spinal Disord 10: 260-271, 1997

19. Nellensteijn J, Ostelo R, Bartels R, Peul W, van Royen B, van Tulder M: Transforaminal endoscopic surgery for symptomatic lumbar disc herniations: A systematic review of the literature. Eur Spine J 19: 181-204, 2010

20. Ruetten S, Komp M: A new full-endoscopic technique for the interlaminar operation of lumbar disc herniations using 6-mm endoscopes: Prospective 2-year results of 331 patients. Minim Invasive Neurosurg 49: 80-87, 2006

21. Ruetten S, Komp M, Merk H: Full-endoscopic interlaminar and transforaminal lumbar discectomy versus conventional microsurgical technique: A prospective, randomized, controlled study. Spine (Phila Pa 1976) 33: 931-939, 2008

22. Ruetten S, Komp M, Merk H: Recurrent lumbar disc herniation after conventional discectomy: A prospective, randomized study comparing full-endoscopic interlaminar and transforaminal versus microsurgical revision. J Spinal Disord Tech 22: 122-129, 2009

23. Smith T, Reeves S, Halsey L, Huber J, Luo J: Effects of BMI on bone loading due to physical activity. J Appl Biomech 3: 1-27, 2017

24. Tittlbach SA, Jekauc D, Schmidt SCE, Woll A, Bös K: The relationship between physical activity, fitness, physical complaints and BMI in German adults - results of a longitudinal study. Eur J Sport Sci 17: 1090-1099, 2017

25. Wahren $\mathrm{H}$ : Herniated nucleus pulposus in a child of twelve years. Acta Orthop Scand 16: 40-42, 1945

26. Yeung AT: Posterolateral endoscopic excision for lumbar disc herniation: Surgical technique, outcome, and complications in 307 consecutive cases. Spine (Phila Pa 1976) 27: 722-731, 2002 